\title{
Community-associated methicillin-resistant Staphylococcus aureus: Implications for the care of children
}

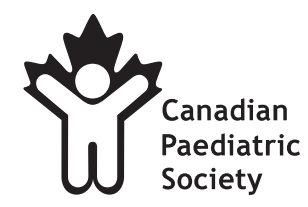

$\mathrm{C}$ anadian guidelines for the prevention and management of community-associated methicillin-resistant Staphylococcus aureus (CA-MRSA) have recently been published (1). These guidelines have been endorsed by the Canadian Paediatric Society.

Until recently, MRSA was found mainly in the hospital setting. Resistance has now appeared in the community as well. CA-MRSA strains appear to have evolved independently of hospital strains and differ from the multiresistant hospital MRSA strains in that CA-MRSA strains tend to be sensitive to a number of non-beta-lactam antibiotics, thus allowing for more treatment options. On the other hand, community strains are often more virulent than hospital strains. In many areas, CA-MRSA strains have acquired the Panton-Valentine leukocidin gene, which confers virulence and has been associated with complicated skin, soft tissue and musculoskeletal infections, and necrotizing pneumonia. It is important to note, however, that not all Panton-Valentine leukocidincontaining $S$ aureus strains are methicillin resistant, and that not all CA-MRSA strains are virulent (2,3). Children appear to be more at risk for CA-MRSA infections than adults.

\section{INVESTIGATION AND TREATMENT OF COMMUNITY-ACQUIRED INFECTIONS}

- If a systemic antibiotic is used, every attempt should be made to obtain material for culture before starting treatment. This includes culturing of skin lesions and aspiration of abscesses, pleural fluid, joint fluid, etc. Culture results will then guide definitive therapy.

- CA-MRSA should be considered in the empirical therapy of any severe life-threatening infection when $S$ aureus is suspected to be involved (eg, necrotizing pneumonia, necrotizing fasciitis, sepsis syndrome). Empirical therapy should include vancomycin in addition to an antistaphylococcal penicillin or cephalosporin, pending culture results.

- For non-life-threatening infections suspected to be due to $S$ aureus, antistaphylococcal penicillins or cephalosporins are still the empirical agents of choice, because the rates of methicillin resistance in Canada are relatively low. CA-MRSA should be considered if there is a poor response to beta-lactam therapy. In populations where more than
$10 \%$ to $15 \%$ of community isolates of S aureus are methicillin-resistant, it may be prudent to consider adding a non-beta-lactam antistaphylococcal antibiotic pending culture results (1).

- Not all skin infections need antibiotic therapy. Minor skin lesions (folliculitis, furuncles and small abscesses) may be managed by hot soaks to promote drainage, or by incision and drainage if pus is present. The use of topical antiseptics may be considered. If a topical antibiotic is deemed necessary, then one that will not be used for systemic therapy, such as bacitracin or mupirocin, should be used, keeping in mind that mupirocin resistance has arisen in some areas of Canada. Systemic antibiotic therapy should be considered if the patient is a newborn or is immunocompromised, or if the skin lesions are rapidly progressing.

- Detailed information on empirical and definitive treatment options for CA-MRSA are presented in the guidelines (1).

\section{PREVENTION}

In the hospital setting, control of MRSA has focused on a 'search and destroy' approach, with widespread screening of potential carriers and extensive use of isolation precautions for those found to be carrying the organism. This approach cannot be extended into the community. Prevention of CA-MRSA must focus on measures to limit transmission of skin infections from person to person regardless of the organism involved. HIV has taught us that blood from any person should be considered as potentially infectious, which led us to the concept of universal precautions. A similar approach is necessary for skin infections. Persons with CA-MRSA are unlikely to be identified unless they have recurrent skin lesions or severe infections. Targeting prevention to those who are known to be infected with CA-MRSA will not be effective and may lead to unwarranted discrimination. The measures presented in the guidelines apply to all presumably infected skin lesions, and should result in control not only of CA-MRSA, but also of virulent strains of methicillin-sensitive $S$ aureus and group A betahemolytic Streptococcus, as well as cutaneous herpes simplex infections and ringworm.

- The skin should be kept clean by regular hand hygiene and regular bathing. 
- Prompt attention should be paid to cuts, abrasions and scratches (clean and cover to protect from external contamination).

- When draining or oozing skin lesions are present:

- Lesions should be covered with appropriate dressings.

- Skin care products (eg, soaps, creams, lotions and cosmetics) should not be shared.

- Towels and other personal items that come in contact with the skin lesions should not be shared until they have been laundered or cleaned.

- Used dressings and other items soiled with wound discharge should be discarded promptly and carefully to avoid exposure of others.

- Hands should be washed after touching any skin lesions or potentially contaminated materials, such as soiled dressings.

- Outside of the acute care setting, there is no indication for the routine screening of contacts of persons with CA-MRSA infection, or for follow-up screening of those with CA-MRSA infections. Screening is indicated only if infections continue to occur in a family setting or any other type of closed population, despite the use of appropriate hygienic measures. Even if CA-MRSA colonization is found, eradication is difficult to achieve and is rarely indicated.

- Prevention also includes avoiding unnecessary or inappropriate use of antibiotics, which may promote proliferation of antibiotic-resistant strains.

- Specific recommendations for daycare centres and schools, sports settings and correctional facilities are presented in the guidelines, as well as considerations for infected pets (1).
- The guidelines also give recommendations for the management of CA-MRSA in the newborn nursery setting. CA-MRSA may be introduced into a nursery from the community by an infected or colonized mother, and spread to her newborn, other newborns or other mothers.

Colonized personnel may also be involved in transmission. Recommendations for control of MRSA in acute care settings have previously been published by the Canadian Paediatric Society (4).

CA-MRSA is here to stay. $S$ aureus is a component of our normal flora, and will evolve, as microbes do, to promote survival of the fittest (5). We simply have to learn how to live with it. As with penicillin-resistant $S$ aureus in the 1950s, and penicillin-resistant pneumococcus in the 1990s, we need to adjust our therapeutic approaches to this new reality. Betalactam antibiotics have been the mainstay of our antibiotic therapy for infections with Gram-positive bacteria since the discovery of penicillin and have served us well for nearly 70 years. We now have to prepare to move on to other classes of antibiotics. As for prevention, good personal hygiene and appropriate care of skin lesions are paramount.

\section{REFERENCES}

1. Barton M, Hawkes, M, Moore D, et al. Guidelines for the prevention and management of community-acquired methicillinresistant Staphylococcus aureus: A perspective for Canadian health care practitioners. Can J Infect Dis Med Microbiol 2006;17(Suppl C):4C-24C.

2. Gillet Y, Issartel B, Vanhems P, et al. Association between Staphylococcus aureus strains carrying gene for Panton-Valentine leukocidin and highly lethal necrotising pneumonia in young immunocompetent patients. Lancet 2002;359:753-9.

3. Martinez-Aguilar G, Avalos-Mishaan A, Hulten K, et al. Community-acquired, methicillin-resistant and methicillinsusceptible Staphylococcus aureus musculoskeletal infections in children. Pediatr Infect Dis J 2004;23:701-6.

4. Canadian Paediatric Society, Infectious Diseases and Immunization Committee [Principal author: D Moore]. Control and treatment of methicillin-resistant Staphylococcus aureus in Canadian paediatric health care institutions. Paediatr Child Health 2006;11:163-65. <www.cps.ca/english/statements/ID/ID06-01.htm> (Version current at March 2, 2007)

5. Nicolle L. Community-acquired methicillin-resistant Staphylococcus aureus: Getting over it. Can J Infect Dis Med Microbiol 2005;16:323-4.

\section{INFECTIOUS DISEASES AND IMMUNIZATION COMMITTEE}

Members: Drs Robert Bortolussi, IWK Health Centre, Halifax, Nova Scotia (chair); Dorothy L Moore, The Montreal Children's Hospital, Montreal, Quebec; Joan Louise Robinson, Edmonton, Alberta; Élisabeth Rousseau-Harsany, Sainte-Justine UHC, Montreal, Quebec (board representative); Lindy Michelle Samson, Children's Hospital of Eastern Ontario, Ottawa, Ontario

Consultant: Dr Noni E MacDonald, IWK Health Centre, Halifax, Nova Scotia

Liaisons: Drs Upton Dilworth Allen, The Hospital for Sick Children, Toronto, Ontario (Canadian Pediatric AIDS Research Group); Scott Alan Halperin, IWK Health Centre, Halifax, Nova Scotia (Immunization Program, ACTive); Charles PS Hui, Children's Hospital of Eastern Ontario, Ottawa, Ontario (Health Canada, Committee to Advise on Tropical Medicine and Travel); Larry Pickering, Centers for Disease Control and Prevention, Atlanta, Georgia (American Academy of Pediatrics, Committee on Infectious Diseases); Marina Ines Salvadori, Children's Hospital of Western Ontario, Ottawa, Ontario (Health Canada, National Advisory Committee on Immunization)

Principal author: Dr Dorothy L Moore, The Montreal Children's Hospital, Montreal, Quebec

The recommendations in this statement do not indicate an exclusive course of treatment or procedure to be followed. Variations, taking into account individual circumstances, may be appropriate. This article also appears in the April 2007 issue of Paediatrics $\mathcal{E}$ Child Health. 


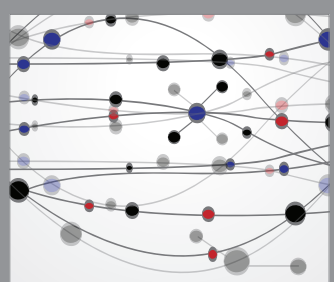

The Scientific World Journal
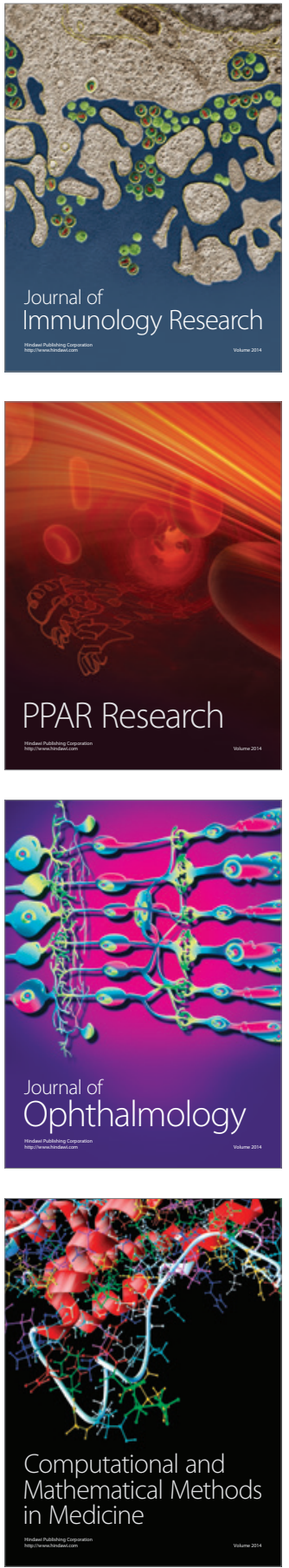

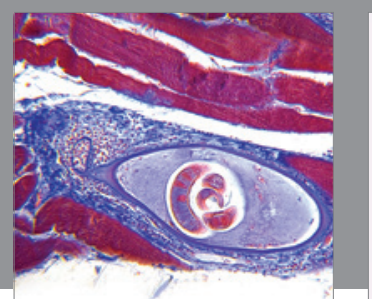

Gastroenterology Research and Practice

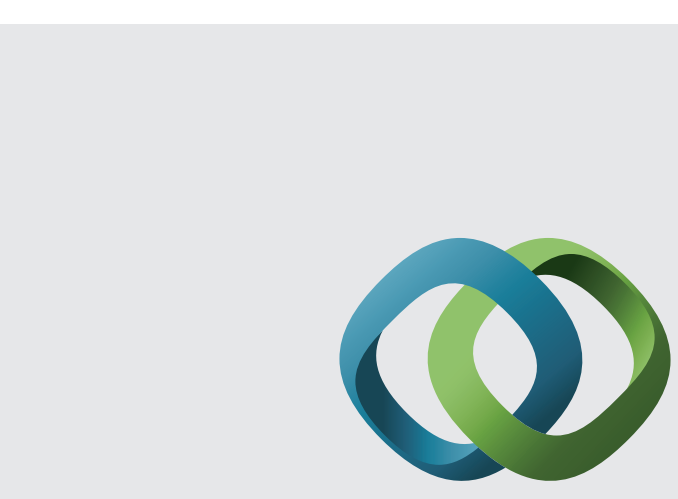

\section{Hindawi}

Submit your manuscripts at

http://www.hindawi.com
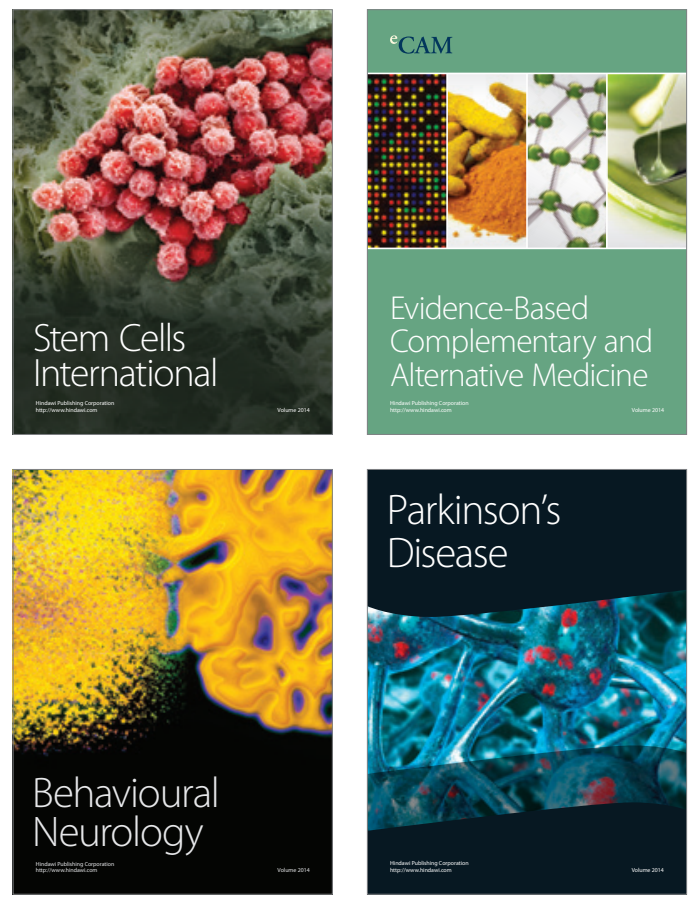
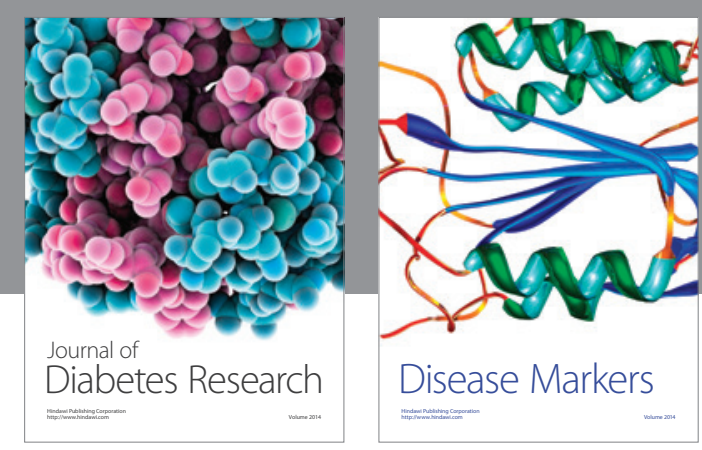

Disease Markers
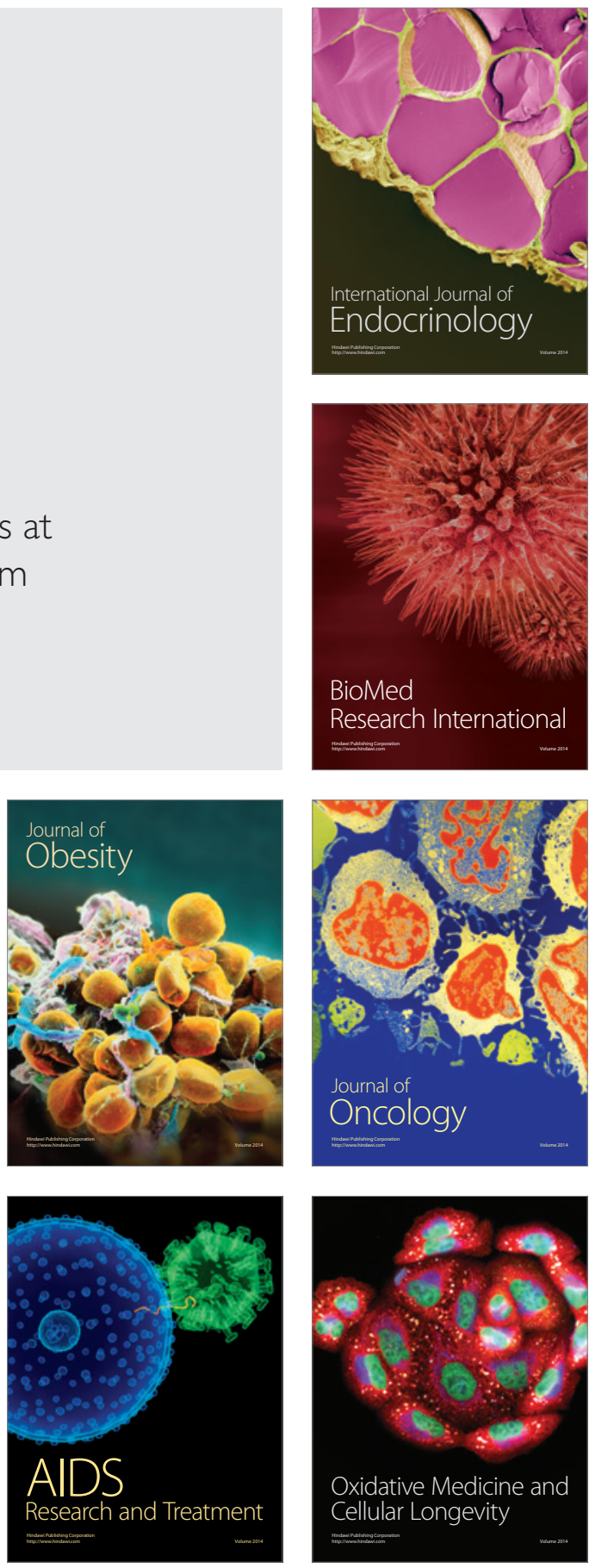